\title{
Reliability of Lattice Gauge Theories
}

\author{
Jad C. Halimeh $\oplus^{1,2,3,4}$ and Philipp Hauke $\oplus^{1,2,3}$ \\ ${ }^{1}$ Kirchhoff Institute for Physics, Ruprecht-Karls-Universität Heidelberg, Im Neuenheimer Feld 227, 69120 Heidelberg, Germany \\ ${ }^{2}$ Institute for Theoretical Physics, Ruprecht-Karls-Universität Heidelberg, Philosophenweg 16, 69120 Heidelberg, Germany \\ ${ }^{3}$ INO-CNR BEC Center and Department of Physics, University of Trento, Via Sommarive 14, I-38123 Trento, Italy \\ ${ }^{4}$ Max Planck Institute for the Physics of Complex Systems, Nöthnitzer Straße 38, 01187 Dresden, Germany
}

(Received 6 February 2020; revised 25 May 2020; accepted 15 June 2020; published 15 July 2020)

\begin{abstract}
Currently, there are intense experimental efforts to realize lattice gauge theories in quantum simulators. Except for specific models, however, practical quantum simulators can never be fine-tuned to perfect local gauge invariance. There is thus a strong need for a rigorous understanding of gauge-invariance violation and how to reliably protect against it. As we show through analytic and numerical evidence, in the presence of a gauge invariance-breaking term the gauge violation accumulates only perturbatively at short times before proliferating only at very long times. This proliferation can be suppressed up to infinite times by energetically penalizing processes that drive the dynamics away from the initial gauge-invariant sector. Our results provide a theoretical basis that highlights a surprising robustness of gauge-theory quantum simulators.
\end{abstract}

DOI: 10.1103/PhysRevLett.125.030503

Introduction.-In modern physics, gauge theories assume a central role, ranging from the standard model of particle physics [1] to emergent exotic solid-state phases $[2,3]$. The defining feature of any gauge theory is its local conservation laws, such as Gauss's law for a U(1) gauge symmetry. Despite their elegance, the computation of gauge theories on classical computers is a daunting task $[4,5]$, particularly for out-of-equilibrium phenomena. Currently, a complementary tool to probe gauge theories is emerging in the form of low-energy tabletop devices, so-called quantum simulators [6-9]. Although still in the developmental phase, these experiments are rapidly advancing [10-18], making one open issue all the more pressing: how can we ensure the reliability of quantum simulators once they scale beyond problems that can be benchmarked by classical computers $[19,20]$ ? For gauge theories, this issue is particularly subtle, since a faithful quantum simulation necessitates not only the correct engineering of the Hamiltonian dynamics but crucially also of the defining local gauge symmetry. It becomes thus an outstanding challenge to understand how gaugeinvariant dynamics may be faithfully simulated-without unrealistically fine-tuned interactions between the constituents of the quantum simulator and in the consequently unavoidable presence of gauge-violating errors.

Published by the American Physical Society under the terms of the Creative Commons Attribution 4.0 International license. Further distribution of this work must maintain attribution to the author(s) and the published article's title, journal citation, and DOI. Open access publication funded by the Max Planck Society.
As we show in this Letter, quantum simulators can reliably reproduce the out-of-equilibrium dynamics of gauge theories even if the prohibitive restriction of perfect gauge invariance is relaxed. Our results are based on numerical studies of $\mathrm{Z}_{2}$ and $\mathrm{U}(1)$ gauge theories as well as analytic proofs. In the presence of gauge invarianceviolating terms, the short-time dynamics deviates only perturbatively slowly from the idealized scenario, and observables reproduce the ideal dynamics during a time frame controlled by the strength of the gauge-violating errors. Upon adding a protection term that energetically penalizes such errors, gauge invariance becomes bounded from above even up to infinite times, cf. Fig. 1. As we explain below and in the Supplemental Material [21], this protection can be mathematically understood as an emergent, deformed gauge symmetry [22]. In surprising contrast to expectations from perturbation theory, our numerics suggests that the strength of the protection term does not need to scale with system size.

Our results thus form a theoretical basis for some previous works, which have found evidence that quantum simulators may approximately retain gauge invariance [23-29], and they complement protection schemes based on classical noise [30]. Moreover, our work complements existing results on equilibrium theories: gauge-invariant equilibrium phases can emerge in a low-energy effective theory, even if the microscopic description breaks gauge invariance, e.g., in topological phases of matter [31], when the gauge degree of freedom decouples because of a large mass [32], or when gauge-noninvariant terms at a small scale renormalize away at large distances ("light from chaos") [33-35]. 


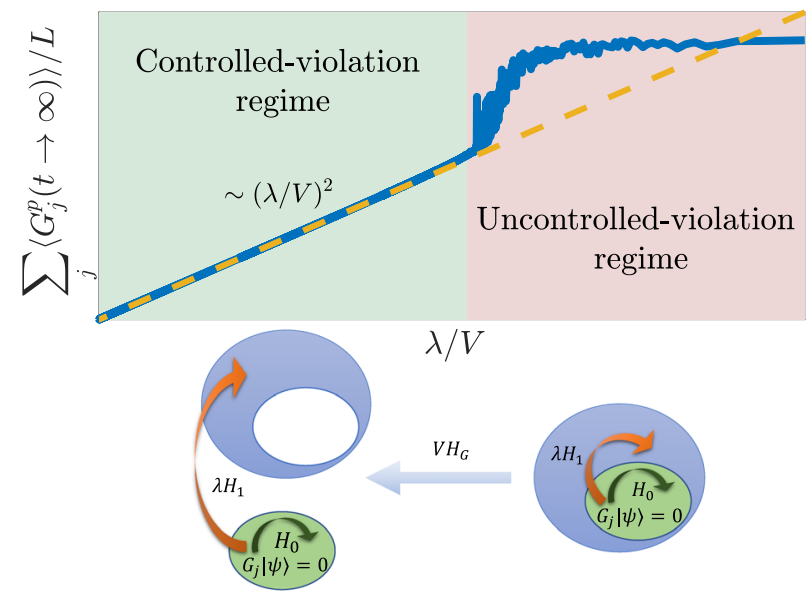

FIG. 1. Right: given a gauge theory with Hamiltonian $H_{0}$ on $L$ matter sites, undesired processes $\propto \lambda H_{1}$ that break gauge symmetry drive the dynamics away from the initial gauge-invariant sector (green bubble) given by the analog of Gauss's law, $G_{j}|\psi\rangle=0 \forall j$ to other sectors in the total Hilbert space (blue bubble). At late times, the average gauge violation assumes a random value. Left: through the introduction of a protection term $\propto V H_{G}$ that energetically penalizes gauge-violating processes, gauge invariance is retained up to infinite times. Two sharply distinct regimes emerge: an uncontrolled-violation regime where $V$ is too small to energetically isolate the initial gauge-invariant sector, and a controlled-violation regime for sufficiently large $V$ where the infinite-time violation scales as $\sim(\lambda / V)^{2}$. The axes are both log scale. See Fig. 4 for a detailed quantitative presentation on $\mathrm{Z}_{2}(p=1)$ and $\mathrm{U}(1)(p=2)$ gauge theories.

Model.-Here, we focus on out-of-equilibrium dynamics, which is highly pertinent for current quantum simulators in ultracold atomic gases [14,16-18] and for which emergent gauge symmetry has received considerably less attention. Although our discussion is general, for concreteness, we consider a $Z_{2}$ gauge theory [36-39] coupled to matter inspired by a recent experiment [16] — we mostly consign similar results for a $U(1)$ gauge theory to the Supplemental Material [21].

The $\mathrm{Z}_{2}$ gauge theory lives on a lattice of $L$ matter sites in one spatial dimension [40], described by the Hamiltonian

$$
H_{0}=\sum_{j=1}^{L}\left[J_{a}\left(a_{j}^{\dagger} \tau_{j, j+1}^{z} a_{j+1}+\text { H.c. }\right)-J_{f} \tau_{j, j+1}^{x}\right],
$$

with periodic boundary conditions. Here, $\tau_{j, j+1}^{\{x, z\}}$ are spin$1 / 2$ Pauli matrices, where the $z(x)$ component stands for the $\mathrm{Z}_{2}$ gauge (electric) field on the link between matter sites $j$ and $j+1$. The matter fields are represented by hardcore bosons with the annihilation operator $a_{j}$ on site $j$. The dynamics of the matter field couples to the $\mathrm{Z}_{2}$ gauge field with strength $J_{a}$. The electric field has energy $J_{f}$.

Gauge invariance is encoded in a set of local symmetry generators

$$
G_{j}=1-(-1)^{j} \tau_{j-1, j}^{x} Q_{j} \tau_{j, j+1}^{x},
$$

with eigenvalues $g_{j}$ and where $Q_{j}=1-2 a_{j}^{\dagger} a_{j}$ is the charge of the matter field. As a $Z_{2}$ lattice equivalent of Gauss's law, the $G_{j}$ commute with the model Hamiltonian $\left[H_{0}, G_{j}\right]=0$, $\forall j$. For a perfectly gauge-invariant system, the Hilbert space thus decouples into different symmetry sectors with fixed local charge $g_{j}=0,2$.

In the standard model of particle physics, such local gauge invariance is postulated at a fundamental level. In a quantum simulator, however, gauge invariance needs to be engineered. Some experiments have used Gauss's law to integrate out either matter or gauge fields, which yields an effective spin theory, suitable for implementation in a quantum computer and with encoded gauge invariance $[10,11,15]$. However, this approach is viable only in one spatial dimension. If, in contrast, both matter and gauge fields are retained as active degrees of freedom, such as in recent ultracold-atom experiments [16-18], exact gauge invariance would require finetuning at unrealistic levels of accuracy. Consequently, such quantum simulators will always have some inherent gauge invariance-breaking processes.

To study the severity of such terms, we add for concreteness the error Hamiltonian

$$
\begin{aligned}
\lambda H_{1}= & \lambda \sum_{j=1}^{L}\left[\left(c_{1} a_{j}^{\dagger} \tau_{j, j+1}^{-} a_{j+1}+c_{2} a_{j}^{\dagger} \tau_{j, j+1}^{+} a_{j+1}+\text { H.c. }\right)\right. \\
& \left.+a_{j}^{\dagger} a_{j}\left(c_{3} \tau_{j, j+1}^{z}-c_{4} \tau_{j-1, j}^{z}\right)\right] .
\end{aligned}
$$

These terms are inspired by Ref. [16], though our conclusions do not depend on their precise form. Here, $\lambda$ is a parameter representing an adjustable gauge-noninvariant error strength and the constants $c_{l}$ are modeled after experimental parameters [21]. Generically, the error term of Eq. (3) will drive the dynamics out of the gauge-invariant subspace, such that after a certain time the expectation value of the Gauss-law operator will be the same as in a random state from the entire Hilbert space (i.e., the space that contains gauge-invariant states as well as all states with $G_{j}|\psi\rangle \neq 0$ ).

Quench dynamics with gauge violation.-We mimic a typical quantum simulator experiment, where the system is initialized in a simple product state $\left|\psi_{0}\right\rangle$ in the gaugeinvariant sector $G_{j}\left|\psi_{0}\right\rangle=0, \forall j$, which is then quenched with the Hamiltonian $H_{0}+\lambda H_{1}$. We choose $\left|\psi_{0}\right\rangle$ such that $\left\langle a_{j}^{\dagger} a_{j}\right\rangle=\left[1+(-1)^{j}\right] / 2$ and $\left\langle\tau_{j, j+1}^{x}\right\rangle=(-1)^{j+1}$, and, following Ref. [16], we set $J_{a}=1$ and $J_{f}=0.54$ (our conclusions do not depend on these precise choices). Our numerical results are obtained using the QUTIP [41,42] and QUSPIN $[43,44]$ exact diagonalization toolkits. For time evolution, we have opted to use our own exact exponentation routine in lieu of these toolkits' time-evolution functions, given the extremely long times we simulate, 
for which solvers based on integrating ordinary differential equations are not optimal.

To evaluate the effect of $H_{1}$ on the gauge-invariant dynamics, we consider the dynamics of the spatiotemporal averages of the violation of Gauss's law,

$$
\varepsilon(t)=\frac{1}{L t} \int_{0}^{t} d s \sum_{j=1}^{L}\left\langle\psi(s)\left|G_{j}\right| \psi(s)\right\rangle
$$

the magnetization in the $x$ direction (the "electric field")

$$
m_{x}(t)=\frac{1}{L t} \int_{0}^{t} d s\left|\sum_{j=1}^{L}\left\langle\psi(s)\left|\tau_{j, j+1}^{x}\right| \psi(s)\right\rangle\right|,
$$

as well as the staggered boson number

$$
N_{\text {stag }}(t)=\frac{1}{L t} \int_{0}^{t} d s\left|\sum_{j=1}^{L}(-1)^{j}\left\langle\psi(s)\left|a_{j}^{\dagger} a_{j}\right| \psi(s)\right\rangle\right| .
$$

In the above, $|\psi(s)\rangle=\exp \left[-i\left(H_{0}+\lambda H_{1}\right) s\right]\left|\psi_{0}\right\rangle$. The respective deviations from the ideal gauge-invariant case are denoted by $\Delta N_{\text {stag }}(t)$ and $\Delta m_{x}(t)$. Further observables are discussed in the Supplemental Material [21]. Note that the gauge violation in Eq. (4) suffices for the $Z_{2}$ gauge theory, but would require an even power in $G_{j}$ or its absolute value for the $\mathrm{U}(1)$ gauge theory [21].

Our numerical results are presented in Fig. 2. The gauge violation grows only gradually, $\varepsilon(t) \sim(\lambda t)^{2}$ at short times, before it saturates at long times. This subleading increase of the gauge violation directly stems from the fact that $\sum_{j} G_{j}$ is gauge invariant and also commutes with $H_{0}$, which necessarily leads to a vanishing first-order contribution in perturbation theory, cf. the Supplemental Material [21]. In contrast, the short-time scaling behavior can change for gauge-invariant observables that do not commute with $H_{0}$, such as $N_{\text {stag }}$ and $m_{x}$. Even though $\Delta m_{x}$ scales as $(\lambda t)^{2}$ at short times, as seen in the inset of Fig. 2(b), $\Delta N_{\text {stag }}$ shown in the inset of Fig. 2(c) scales instead as $\lambda t^{2}$, with the latter emenating from the first-order term in perturbation theory, cf. the Supplemental Material [21]. Importantly, $N_{\text {stag }}(t)$ remains nevertheless close to the gauge-invariant dynamics up to $t \approx 1 / \lambda$. Thus, there is a clear time frame over which gauge-noninvariant terms do not compromise observable properties, and by decreasing $\lambda$ this time frame can be improved in a controlled manner.

Quench dynamics with energy protection.-Some promising proposals have suggested to perturbatively generate the desired gauge-theory Hamiltonian by adding a term proportional to Gauss's law, which energetically penalizes violations of gauge invariance $[7,8,24]$. A similar term has been discussed for protecting stored quantum information [45]. We now systematically address the question of how
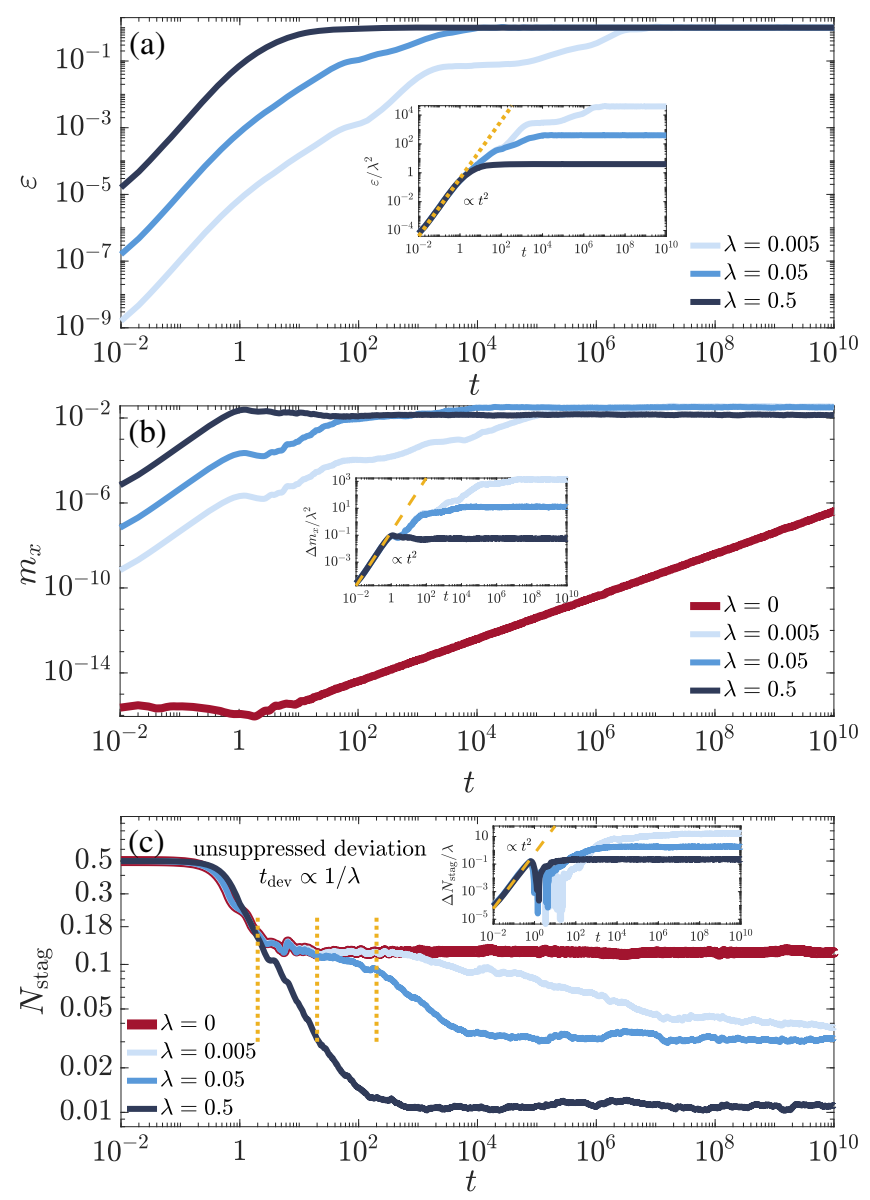

FIG. 2. Effect of gauge invariance-breaking terms on the dynamics of a $Z_{2}$ gauge theory. Spatiotemporal averages of (a) the gauge-invariance violation in Eq. (4), (b) the electric field in Eq. (5), and (c) the staggered boson number in Eq. (6), deviate only gradually from the ideal dynamics, before gauge-noninvariant effects begin to dominate after a timescale of $t_{\mathrm{dev}} \propto 1 / \lambda$. Insets: rescaled deviations from ideal dynamics, showing the perturbative growth with $\lambda$. Data for $L=6$ matter sites. See the Supplemental Material [21] for further information.

such a term restores the ability to quantum simulate gaugeinvariant dynamics.

To analyze this scenario, we introduce a protection term

$$
V H_{G}=V \sum_{j} G_{j}
$$

which energetically penalizes all violations of $G_{j}\left|\psi_{0}\right\rangle=0$, with adjustable protection strength $V$. Note that Eq. (7) is not sufficient for the $\mathrm{U}(1)$ gauge theory, and should rather be $V H_{G}=V \sum_{j} G_{j}^{2}$ [21]. Our quench Hamiltonian is thus given by $H=H_{0}+\lambda H_{1}+V H_{G}$. Consequently, in Eqs. (4), (5), and (6) we now have $|\psi(s)\rangle=\exp \left[-i\left(H_{0}+\lambda H_{1}+\right.\right.$ $\left.\left.V H_{G}\right) s\right]\left|\psi_{0}\right\rangle$. In Fig. 3, we show the effect of $V$ on $\varepsilon(t)$, $\Delta N_{\text {stag }}(t)$, and $\Delta m_{x}(t)$. As can be shown in degenerate perturbation theory [21], the violation $\varepsilon(t)$ of Gauss's law is suppressed by $(\lambda / V)^{2}$ at sufficiently large $V$, cf. the 

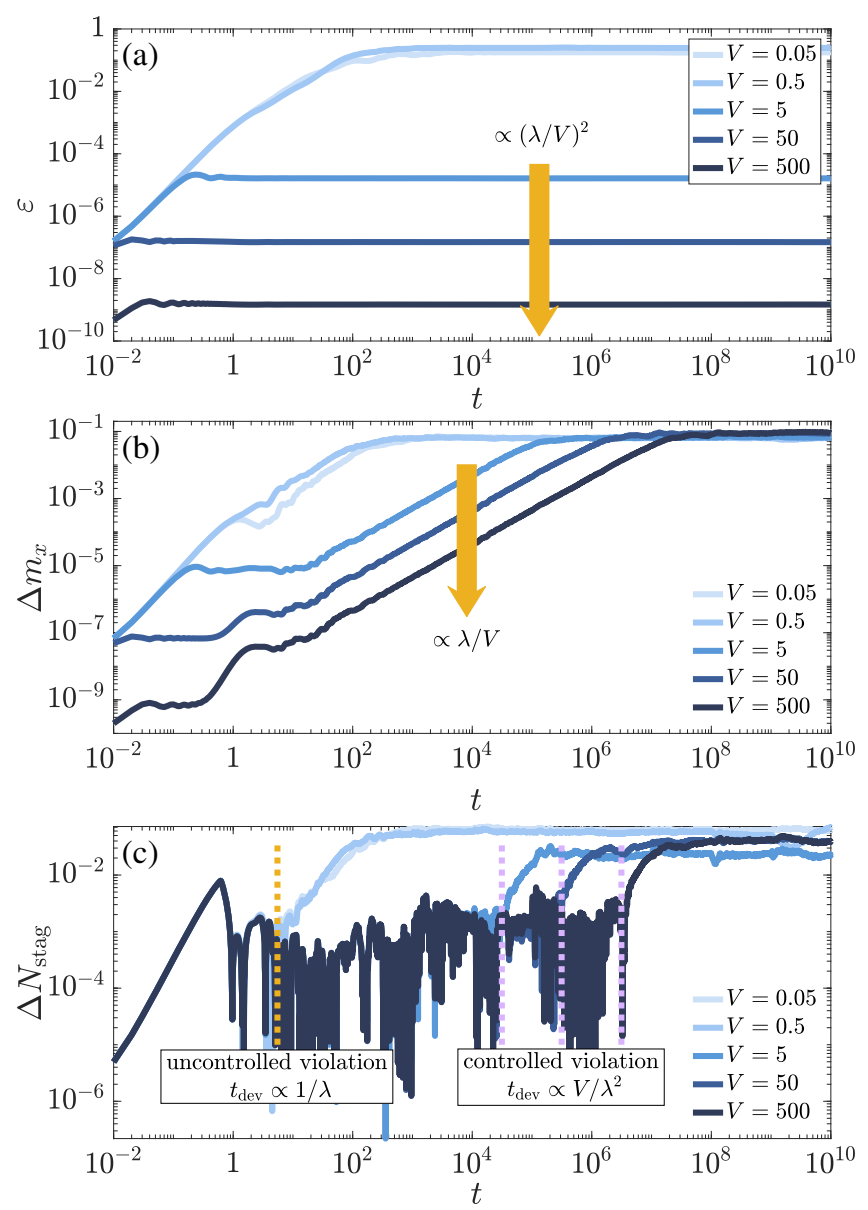

FIG. 3. Dynamics of the spatiotemporal averages of (a) the gauge-invariance violation in Eq. (4), (b) the electric field in Eq. (5), and (c) the staggered boson number in Eq. (6) at error strength $\lambda=0.05$ and at various values of the protection strength $V$ for $L=6$ matter sites. We see two clear regimes: at small $V$ the gauge-noninvariant behavior dominates after a timescale $\propto 1 / \lambda$. When $V$ is sufficiently large (controlled-violation regime), the gauge-invariance violation in (a) is indefinitely suppressed by $(\lambda / V)^{2}$, while deviations in the electric field in (c) are suppressed by $\lambda / V$ up to a timescale $\propto V / \lambda^{2}$. The deviation in the staggered boson number becomes uncontrolled only at times beyond $t \propto V / \lambda^{2}$. See the Supplemental Material [21] for corresponding results at $\lambda=0.005$ and 0.5 .

Supplemental Material [21]. One might suspect that such an energy penalty would only suppress gauge-noninvariant processes at short times before the system eventually accesses all possible gauge-invariant sectors at long times. Interestingly, however, once $V$ is on the order of a few $\lambda$, a "controlled-violation" regime is reached, where $\varepsilon(t)$ is protected for all simulated times (which are many orders of magnitude larger than what is reachable in current experiments).

In contrast, for sufficiently large $V$ the deviation of $m_{x}(t)$ is suppressed only by $\lambda / V$, as shown in Fig. 3(b), with the suppression ceasing at a timescale $\propto V / \lambda^{2}$, cf. the Supplemental Material [21]. At the same timescale, $N_{\text {stag }}$
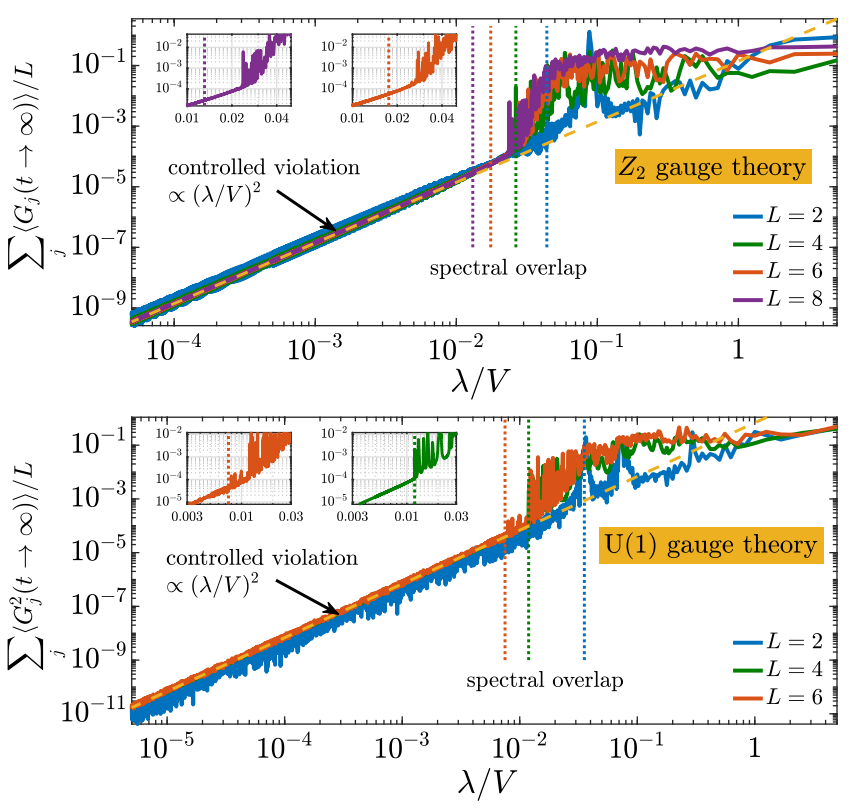

FIG. 4. Infinite-time violation $\sum_{j}\left\langle G_{j}(t \rightarrow \infty)\right\rangle / L$ in the $\mathrm{Z}_{2}$ gauge theory (top panel) and $\sum_{j}\left\langle G_{j}^{2}(t \rightarrow \infty)\right\rangle / L$ in the $\mathrm{U}(1)$ gauge theory (bottom panel) as a function of $\lambda / V$ for $\lambda=0.05$, computed for several system sizes $L$. We see two separate regimes where at large $V$ the violation is controlled and scales as $(\lambda / V)^{2}$, while at small $V$ the violation is uncontrolled. The dotted lines indicate the largest $V$ at which there is an energy overlap between the initial gauge-invariant sector, and other symmetry sectors, which we protect against. In the controlledviolation regime, the violation is system size-independent. The vertical dotted lines correspond to the largest value of $V$ (for a given system size $L$ ) at which the initial gauge-invariant sector still has spectral overlap with other sectors.

starts deviating from the gauge-invariant case if the values of $V$ lie in the controlled-violation regime, see Fig. 3(c). Furthermore, the terms that drive these deviations can be described as gauge-invariant processes in degenerate perturbation theory, and could thus be absorbed into a renormalized, gauge-invariant $H_{0}$. See the Supplemental Material [21] for more details. The existence of these timescales is a considerable improvement, since outside the controlled-violation regime deviations from the ideal gauge-invariant dynamics proliferate already at an earlier timescale $\propto 1 / \lambda$.

Physically, $V$ protects gauge invariance by opening a large energy gap between the sector $G_{j}|\psi\rangle=0, \forall j$, and all other sectors. Since the bandwidth of each sector increases with system size, one could expect the necessity to scale $V$ with $L$, which would invalidate this protection mechanism for large-scale quantum simulators. However, our numerics suggest that this is not the case. We illustrate this in Fig. 4 for the infinite-time per-site violation of Gauss's law as a function of $\lambda / V$ (with fixed $\lambda=0.05$ ). We see two clear regimes. The first at small values of $V$ shows an uncontrolled violation that heavily depends on how the different 
gauge-invariant sectors are coupled to one another. The second regime, however, displays a controlled violation that scales as $(\lambda / V)^{2}$. This regime is expected once the gauge-invariant sector we start in is energetically well separated from other sectors, as can be shown in degenerate perturbation theory and made mathematically rigorous by adapting the results of Ref. [22] (see the Supplemental Material [21]). For any unitary symmetry that is broken on a scale $\sim \lambda$, the opening of a gap generates an emergent symmetry that is perturbatively in $(\lambda / V)^{2}$ close to the original one. Surprisingly, however, the scaling as $(\lambda / V)^{2}$ sets in much before full separation between sectors is achieved. This onset appears to be largely independent of system size, contrary to the analytic arguments based on perturbation theory and the emergent gauge symmetry (see the Supplemental Material [21]). The reason is that the relevant gap to gauge-violating sectors is not to be counted relative to the entire gauge-invariant sector, but only to the energy region that is populated during the quench. A similar effect has been observed for the robustness of the ground-state degeneracy in topological matter [31].

Our results in Figs. 3 and 4 illustrate how once the controlled-violation regime is reached at a sufficiently large $V$, extrapolations to the ideal case become possible in both the gauge-invariance violation and gauge-invariant observables. Indeed, Fig. 3 shows a clear timescale before which deviations in a gauge-invariant observable are well-determined as a function of $\lambda$ and $V$. Even better, in this regime the control in the gauge-invariance violation is not limited by any timescale, but rather persists indefinitely $\propto(\lambda / V)^{2}$.

Conclusions and outlook.-We have carried out a thorough analysis, through exact diagonalization and perturbation theory, of the reliability of lattice gauge theories in out-of-equilibrium dynamics. We have found that small gauge-nonivariant processes (of strength $\lambda$ ) do not compromise the desired dynamics of observables up to a clear time frame $\sim \lambda^{-1}$. Moreover, when introducing a sufficiently expensive energy penalty of strength $V$ for such processes, the gauge-invariance violation enters a controlled-violation regime where it scales as $(\lambda / V)^{2}$, up to infinite times, and is also robust with respect to system size.

The suppression in observables' deviations from their gauge-invariant dynamics presents a more varied picture. This is to be expected because when a gauge theory is broken by a small parameter, another gauge theory perturbatively close to it emerges [22] that, even though gaugeinvariant, is still different from its initial counterpart. The original theory's exact dynamics can, however, be recovered by an appropriate absorption of the new terms into renormalized parameters [33,34].

In state-of-the-art quantum simulators based on ultracold atoms and trapped ions, gauge-invariance breakings as low as $\lambda=\mathcal{O}\left(0.5 J_{a}\right)$ [16] and in specific cases protection strengths as large as $V=\mathcal{O}(3-28 \lambda)[18,24]$ can already be reached. These are very encouraging results for experimental efforts as they indicate that introducing an energy penalty leads to an indefinite protection of gauge invariance, with energy scales that are achievable in current setups. Even more, once the protected regime is reached, a well-defined extrapolation becomes possible from gauge invariance-violating data to a perfect gauge theory.

The authors are grateful to Z. Jiang and G. Morigi for useful comments, and to J. Berges, F. Jendrzejewski, R. Ott, B. Yang, and T. Zache, for stimulating discussions and collaboration on related work. J. C. H. thanks J. C. Louw for interesting discussions related to exact diagonalization. This work is part of and supported by the DFG Collaborative Research Centre SFB 1225 (Grant No. ISOQUANT), the Provincia Autonoma di Trento, and the ERC Starting Grant StrEnQTh (Project-ID 804305).

[1] T. Cheng and L. Li, Gauge Theory of Elementary Particle Physics, Oxford Science Publications (Clarendon Press, New York, 1984).

[2] L. Balents, Nature (London) 464, 199 (2010).

[3] L. Savary and L. Balents, Rep. Prog. Phys. 80, 016502 (2016).

[4] C. Gattringer and C. Lang, Quantum Chromodynamics on the Lattice: An Introductory Presentation, Lecture Notes in Physics (Springer, Berlin, Heidelberg, 2009).

[5] E. Calzetta and B. Hu, Nonequilibrium Quantum Field Theory, Cambridge Monographs on Mathematical Physics (Cambridge University Press, New York, 2008).

[6] U.-J. Wiese, Ann. Phys. (Amsterdam) 525, 777 (2013).

[7] E. Zohar, J. I. Cirac, and B. Reznik, Rep. Prog. Phys. 79, 014401 (2015).

[8] M. Dalmonte and S. Montangero, Contemp. Phys. 57, 388 (2016).

[9] M. C. Bauls, R. Blatt, J. Catani, A. Celi, J. I. Cirac, M. Dalmonte, L. Fallani, K. Jansen, M. Lewenstein, S. Montangero, C. A. Muschik, B. Reznik, E. Rico, L. Tagliacozzo, K. Van Acoleyen, F. Verstraete, U.-J. Wiese, M. Wingate, J. Zakrzewski, and P. Zoller, arXiv:1911.00003.

[10] E. A. Martinez, C. A. Muschik, P. Schindler, D. Nigg, A. Erhard, M. Heyl, P. Hauke, M. Dalmonte, T. Monz, P. Zoller, and R. Blatt, Nature (London) 534, 516 (2016).

[11] H. Bernien, S. Schwartz, A. Keesling, H. Levine, A. Omran, H. Pichler, S. Choi, A. S. Zibrov, M. Endres, M. Greiner, V. Vuletić, and M. D. Lukin, Nature (London) 551, 579 (2017).

[12] H.-N. Dai, B. Yang, A. Reingruber, H. Sun, X.-F. Xu, Y.-A. Chen, Z.-S. Yuan, and J.-W. Pan, Nat. Phys. 13, 1195 (2017).

[13] N. Klco, E. F. Dumitrescu, A. J. McCaskey, T. D. Morris, R. C. Pooser, M. Sanz, E. Solano, P. Lougovski, and M. J. Savage, Phys. Rev. A 98, 032331 (2018).

[14] F. Görg, K. Sandholzer, J. Minguzzi, R. Desbuquois, M. Messer, and T. Esslinger, Nat. Phys. 15, 1161 (2019).

[15] C. Kokail, C. Maier, R. van Bijnen, T. Brydges, M. K. Joshi, P. Jurcevic, C. A. Muschik, P. Silvi, R. Blatt, C. F. Roos, and P. Zoller, Nature (London) 569, 355 (2019). 
[16] C. Schweizer, F. Grusdt, M. Berngruber, L. Barbiero, E. Demler, N. Goldman, I. Bloch, and M. Aidelsburger, Nat. Phys. 15, 1168 (2019) ..

[17] A. Mil, T. V. Zache, A. Hegde, A. Xia, R. P. Bhatt, M. K. Oberthaler, P. Hauke, J. Berges, and F. Jendrzejewski, arXiv:1909.07641.

[18] B. Yang, H. Sun, R. Ott, H.-Y. Wang, T. V. Zache, J. C. Halimeh, Z.-S. Yuan, P. Hauke, and J.-W. Pan (to be published).

[19] P. Hauke, F. M. Cucchietti, L. Tagliacozzo, I. Deutsch, and M. Lewenstein, Rep. Prog. Phys. 75, 082401 (2012).

[20] J. Berges, Nature (London) 569, 339 (2019).

[21] See the Supplemental Material at http://link.aps.org/ supplemental/10.1103/PhysRevLett.125.030503 for a derivation of the emergent deformed gauge symmetry due to the violation of the original one, results repeated for a $\mathrm{U}(1)$ gauge theory (where the main conclusions remain unchanged with respect to the $\mathrm{Z}_{2}$ gauge theory), a derivation of the constants $c_{l}, l \in\{1,2,3,4\}$, further supporting results for the $Z_{2}$ gauge theory, a presentation of the detailed derivation in perturbation theory of the short-time dynamics due to a gauge invariance-breaking term of strength $\lambda>0$, and a presentation of the detailed derivation in perturbation theory of the effective Hamiltonian due to introducing an energy-penalty term with strength $V$ to protect against the gauge invariance-breaking processes.

[22] C. T. Chubb and S. T. Flammia, J. Math. Phys. (N.Y.) 58, 082202 (2017).

[23] D. Banerjee, M. Dalmonte, M. Müller, E. Rico, P. Stebler, U.-J. Wiese, and P. Zoller, Phys. Rev. Lett. 109, 175302 (2012).

[24] P. Hauke, D. Marcos, M. Dalmonte, and P. Zoller, Phys. Rev. X 3, 041018 (2013).

[25] S. Kühn, J. I. Cirac, and M.-C. Bañuls, Phys. Rev. A 90, 042305 (2014).

[26] Y. Kuno, K. Kasamatsu, Y. Takahashi, I. Ichinose, and T. Matsui, New J. Phys. 17, 063005 (2015).
[27] Y. Kuno, S. Sakane, K. Kasamatsu, I. Ichinose, and T. Matsui, Phys. Rev. D 95, 094507 (2017).

[28] A. S. Dehkharghani, E. Rico, N. T. Zinner, and A. Negretti, Phys. Rev. A 96, 043611 (2017).

[29] J. C. P. Barros, M. Burrello, and A. Trombettoni, arXiv: 1911.06022.

[30] K. Stannigel, P. Hauke, D. Marcos, M. Hafezi, S. Diehl, M. Dalmonte, and P. Zoller, Phys. Rev. Lett. 112, 120406 (2014).

[31] M. B. Hastings and X.-G. Wen, Phys. Rev. B 72, 045141 (2005).

[32] C. Wetterich, Nucl. Phys. B915, 135 (2017).

[33] D. Foerster, H. Nielsen, and M. Ninomiya, Phys. Lett. 94B, 135 (1980).

[34] E. Poppitz and Y. Shang, Int. J. Mod. Phys. A 23, 4545 (2008).

[35] M. Golterman, Nucl. Phys. B94, 189 (2001).

[36] E. Zohar, A. Farace, B. Reznik, and J. I. Cirac, Phys. Rev. Lett. 118, 070501 (2017).

[37] L. Barbiero, C. Schweizer, M. Aidelsburger, E. Demler, N. Goldman, and F. Grusdt, Sci. Adv. 5 (2019) https://doi.org/ 10.1126/sciadv.aav7444.

[38] J. Frank, E. Huffman, and S. Chandrasekharan, Phys. Lett. B 806, 135484 (2020).

[39] U. Borla, R. Verresen, F. Grusdt, and S. Moroz, Phys. Rev. Lett. 124, 120503 (2020).

[40] Although Ref. [16] considers a minimal model, we are interested here in gauge invariance in extended systems.

[41] J. Johansson, P. Nation, and F. Nori, Comput. Phys. Commun. 183, 1760 (2012).

[42] J. Johansson, P. Nation, and F. Nori, Comput. Phys. Commun. 184, 1234 (2013).

[43] P. Weinberg and M. Bukov, SciPost Phys. 2, 003 (2017).

[44] P. Weinberg and M. Bukov, SciPost Phys. 7, 20 (2019).

[45] Z. Jiang and E. G. Rieffel, Quantum Inf. Process. 16, 89 (2017). 